\title{
Physiochemical Characteristics and Heavy Metal Levels of Water from Hand Dug Wells in Ikiran-Ile, Akoko Edo Local Government Area, Edo State, Nigeria
}

*MAJU-OYOVWIKOWHE, GE; SHUAIB, II

\author{
Department of Geology, University of Benin, Benin City. Nigeria \\ *Corresponding Author Email: efetobore.maju@uniben.edu
}

\begin{abstract}
Groundwater is water beneath the surface of the earth. The primary source is precipitation from rain, snow, and hail. Groundwater commonly occurs as water that fills pore spaces between mineral or rock grains in sediments and sedimentary rocks. This study is to evaluate the physiochemical characteristics and selected heavy metal levels of Water from Hand Dug Wells in Ikiran-Ile in Akoko Edo Local Government Area of Edo State, Nigeria. A total of twenty (20) water samples were collected in very clean containers from twenty (20) locations in Ikiran-Ile village, and taken to the laboratory immediately for physical, chemical and heavy metal analysis using standard laboratory techniques. Results from the study, shows that the ground water is odorless and tasteless, soft to moderately hard, contains free carbon oxide and alkalinity is due to bicarbonate ions. Sodium (Na), chloride $(\mathrm{Cl})$, Iron $(\mathrm{Fe})$ bicarbonates $\left(\mathrm{HCO}_{3}\right)$ calcium $\left(\mathrm{Ca}^{2+}\right)$ potassium $\left(\mathrm{K}^{+}\right)$, and Magnesium $\left(\mathrm{Mg}^{2+}\right)$ are the most abundant of the dissolved cations in the water, while lead $(\mathrm{Pb})$, chromium $(\mathrm{Cr})$ and zinc $(\mathrm{Zn})$ have high concentration levels. These cations exceeded the W.H.O recommended limits. Dissolved solids in the samples are below recommended maximum limits by W.H.O. Samples also showed high bacteria load which primarily originated from effluent discharge from pit toilets and waste dumps and can constitute health risk on ingestion. Highly populated areas have groundwater with high concentration of dissolved ions and the converse for areas of low population. From the results of the analysis, conclusion is drawn to the fact that, the groundwater chemistry in the study area has been influenced greatly by human activities than the bedrock geology of the area. It is therefore recommended that well location should be at least, $30 \mathrm{~m}$ away from any source of contamination such as soak-ways, adequate treatment must be given to well water such as chlorination, boiling to destroy any bacteria and filtration to remove all unwanted/undesirable constituents that may be present, and a policy on land use planning and urban development to guard against indiscriminate setting of wells within the centre of the village should be established. This study has been able to enhance sustainable water development, since the health of inhabitants in a community depends to a large extent on the quality of groundwater supply.
\end{abstract}

\section{DOI: https://dx.doi.org/10.4314/jasem.v23i2.13}

Copyright: Copyright (C 2019 Maju-Oyovwikowhe and Shuaib. This is an open access article distributed under the Creative Commons Attribution License (CCL), which permits unrestricted use, distribution, and reproduction in any medium, provided the original work is properly cited.

Dates: Received: 30 December 2018; Revised: 24 January 2019; Accepted 29 January 2019

Keywords: Groundwater, Physiochemical, Heavy metals, Ikiran-Ile

The term "groundwater" can be described as the water beneath the ground surface; in pore spaces in sedimentary rocks and/or in the fractures in basement rocks. Groundwater is one of the reservoirs of the hydrologic cycle. The study of groundwater occurrences and its movement has become very important in view of acute shortage of pipe borne water supply and surface water in many places. The study area is Ikiran-Ile and it is part of the Southwestern Basement Complex of Nigeria. The major sources of water in the area include hand-dug wells and streams. However, human activities in the area have adversely polluted the water bodies and have resulted in outbreak of diseases among the residents. Groundwater pollution can be seen as any detrimental alteration of the physiochemical and biological qualities of water by man and/or by a natural processes such as volcanic eruption, earthquake etc. This makes the water less suitable or unfit for plant and animal use (including human) than its original state. Such environmental pollution includes contamination of water through air or land quantities which greatly affect their beneficial use. According to Walton (1970), pathogenic bacteria, viruses and other micro-organisms are rarely found in Hydrospheric water since they do not multiply. They eventually die in the subsurface environment except in poorly constructed wells that let in biological contamination. From the earliest times, mankind has relied heavily on water supply from hand-dug wells for their domestic, agricultural and industrial uses. Over $80 \%$ of the population of inhabitants in Ikiran-Ile village and its environs derive their water supply by harnessing hand-dug well waters. Hence, it is very important that proper measures be taken to avoid groundwater contamination and to enhance sustainable water development, since the health of inhabitants in a community depends to a large extent on the quality of groundwater supply. Water quality entails a proper evaluation of its biological, chemical and physical properties. Therefore, the quality of water for various purposes depends on the concentration of these substances. The objective of this study is to evaluate the physiochemical characteristics and selected heavy metal levels of Water from Hand Dug Wells in Ikiran-Ile in Akoko Edo Local Government Area of Edo State, Nigeria, in order to educate the inhabitants of the area on any 
impending health danger in connection with the domestic use of these waters. Akujieze (1999).

\section{MATERIALS AND METHODS}

Description of study area: The study area is Ikiran-Ile and is located in Akoko Edo Local government area of Edo state. Its co-ordinate is $\mathrm{N} 07^{\circ} 25^{\prime} 11.4^{\prime \prime} \mathrm{E}$ $006^{\circ} 01$ ' $16.7^{\prime \prime}$

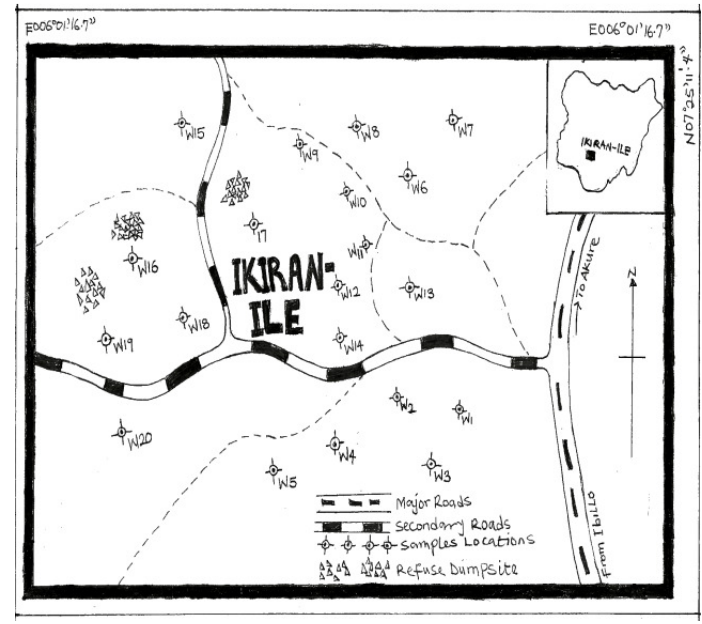

Fig 1:Sketched map of Ikiran-Ile showing sampled locations

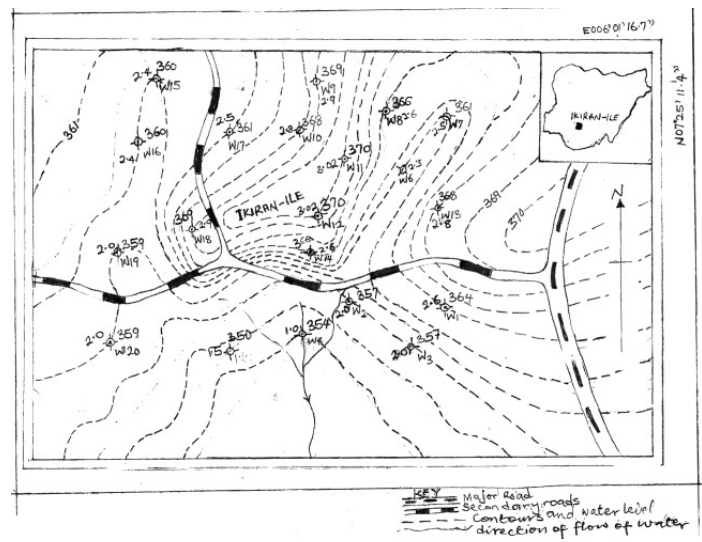

Fig 2: Sketched Contour Map of Ikiran-Ile showing Elevation Wells and Water Level

General geology of the area: Ikiran-Ile is located at the Northern-Western part of Edo state; it is part of the basement complex of South-Western Nigeria. Rahman, (1976), reported that major groups of rocks can be recognized in the area and these includemigmatite-gneiss complex rock which comprises biotite and biotite hornblende gneiss, quartzites and quartz schist and small lenses of calcsilicate' migmatized to unmigmatizedparaschists and meta igneous rock which consist of peliticschists, quartzitesamphibolites, arkose rocks, metaconglomerates, marbles and calc-silicate rocks, charnockitic rocks; older granites which comprise rocks and potassicsyenite; unmetamorphosed dolerite dyke believed to be the youngest. The rocks of the study area were affected by the pan African Orogeny which occurred between 700-750 Ma (Oloto and Anyanwu 2013). Two phases of folding believed to be related to the older granite orogeny (Pan- African) have been described affecting the gneiss-migmatite quartzite complex and the slightly migmatised to unmigmatisedschists and meta-igneous rock. The first folds (f1) are reclined in style (f2) have forms that are variable from open to isoclinal with steep to vertical $\mathrm{N}-\mathrm{S}$ trending axial planes. A barrovian type metamorphism has affected the area and metamorphic grade is from green schist to amphibolitesfacies. Three episodes of metamorphism have been recognized in the peliticschists, the first (Ml) is related to f1 deformation, the second (M2) metamorphism developed during a static phase following f1 and the third (M3) metamorphism which reached a climax in the amphibolitesfacies started during F3 determination and out lasted it.

Sample collection and preservation: A total of twelve (12) samples were collected from twelve (12) different locations in Ikiran-Ile village for this study. Based on the scope of this work, all the water samples used were collected and taken to the laboratory immediately.

Depth to groundwater: The depth to groundwater varies from $2.50 \mathrm{~m}$ to $5.00 \mathrm{~m}$ which is relatively shallow. The shallowness of the groundwater is disadvantageous because it renders the groundwater system highly susceptible to contamination by anthropogenic sources. The rainwater would not have had enough time to percolate through a sizeable soil column that will do the purification of the water before storage in the aquifer.

Methods: Conductivity and temperature values of the water samples were measured with an instrument known as HANNA conductivity and temperature meter EC/TDS. ${ }^{\circ} \mathrm{C} /{ }^{\circ} \mathrm{F}$. Water proof $\mathrm{H} 198312$. The turbidity was determined with turbidity meter wagWT 3020, water proof with serial number 197619 and parameters such as $\mathrm{NO}_{3}{ }^{-}, \mathrm{Fe}^{2+}, \mathrm{Na}^{+}, \mathrm{Mg}^{2+}$ and $\mathrm{Ca}^{2+}$ were analyzed using a spectrophotometer. Wagtech photometer with wavelength ranging from 410-640 and serial number $3400-008$ was also used to determine parts of the chemical parameters. Physical parameters like odour, taste, and colour were observed in the field, at the site of collection. The alkalinity, hardness and the analysis of unstable radicals like nitrate and bicarbonate were all carried out shortly after collection of water sample. In the case of colour, a visual observation of water sample 
was done and the results were recorded as clear, slightly clear, yellowish and slightly yellowish

Determination of hardness: Hardness of water is determined by taking $50 \mathrm{~m} 1$ sample into a $125 \mathrm{~m} 1$ Erlemeyer flask. Add 1-2m1 buffer, add 1-2ml drops of Erichrome Black $\mathrm{T}$ and titrate slowly stirring continuously until the last reddish tinge disappears from the solution (endpoint). The addition of the last few drops should be at 3-5sec interval. Magnesium hardness was subtracted from the total hardness to obtain the calcium hardness.

Determination of conductivity: The conductivity was determined directly from the various samples by the HANNA conductivity meter. Conductivity is another important criterion in measuring the electrical resistance of a solution which is to draw information on the totalionic concentration in the solution. The conductivity of water is related to the total concentration of dissolved ions, their valences, mobility and temperature. The purity of water is commonly checked by conductivity meter.

Total dissolved solids (TDS): 100ml of the samples were measured and filtered into $100 \mathrm{ml}$ measuring cylinder using a pre-weighed filter paper; it was made up to $100 \mathrm{ml}$ with distilled water and later transferred to a pre-weighed crucible and evaporated into dryness in steam water bath, dried in an oven at $180^{\circ} \mathrm{C}$ for $1 \frac{1}{2}$ hours, cooled in a desiccator and re-weighed. The weight of the crucible was recorded as $\mathrm{W}_{1}$ and dissolved solid as $\mathrm{W}_{2}$.

$\operatorname{TDS}(\mathrm{mg} / \mathrm{L})=\mathrm{W}_{2-} \mathrm{W}_{1} \times 10^{3}$.

The total alkalinity was determined by titration with a standard solution. $100 \mathrm{ml}$ of unfiltered water sample was pipetted into $250 \mathrm{ml}$ beaker. Three (3) drops of phenolphthalein indicator was added to check for presence of carbonate and hydroxide alkalinities at the initial stage of the titration. There was no change in coloration of the samples. The 3 drops of methyl orange indicator was added and titrated further until the colour changed from yellow to brick red, the value was recorded.

Total suspended solids (TSS): This represents the portion of solid retained by a filter. The pre-weighed filter paper used for total dissolved solids was dried at $105^{\circ} \mathrm{C}$. This was transferred into an oven at $105^{\circ} \mathrm{C}$ for $1 \frac{1}{2}$ hours it was cooled in a desiccator and weighed. The weight of pre-weighed filter paper was $\mathrm{W}_{1}$ and the weight of filter paper and residue after drying was $\mathrm{W}_{2}$.

$\operatorname{TSS}\left(\mathrm{mg} / \mathrm{L}=\mathrm{W}_{2}-\mathrm{W}_{1} \times 10^{3}\right.$

\begin{tabular}{|c|c|c|c|c|c|c|c|c|c|c|c|c|c|}
\hline $\mathbf{S} / \mathbf{N}$ & Parameters/Unit & W1 & W2 & W3 & W4 & W5 & W6 & W7 & W8 & W9 & W10 & W11 & W12 \\
\hline 1 & APP (CLEAR) & $\mathrm{C}$ & $\mathrm{C}$ & $\mathrm{C}$ & $\mathrm{C}$ & $\mathrm{C}$ & $\mathrm{C}$ & $\mathrm{C}$ & $\mathrm{C}$ & $\mathrm{C}$ & SC & $\mathrm{SC}$ & $\mathrm{C}$ \\
\hline 2 & DWW (m) & 2.60 & 2.00 & 2.00 & 1.80 & 1.90 & 2.30 & 2.50 & 2.60 & 2.90 & 2.80 & 3.02 & 3.02 \\
\hline 3 & Temp. (NS) & 28.04 & 28.7 & 28.4 & 27.9 & 28.4 & 28.0 & 28.3 & 27.9 & 28.1 & 27.8 & 27.7 & 28.1 \\
\hline 4 & Cond. $(\mu \mathrm{s} / \mathrm{cm})$ & 170 & 40 & 180 & 200 & 180 & 160 & 50 & 70 & 120 & 210 & 90 & 60 \\
\hline 5 & $\mathrm{pH}$ & 7.33 & 7.10 & 6.97 & 6.95 & 7.12 & 7.3 & 6.90 & 6.98 & 6.89 & 7.5 & 7.8 & 7.8 \\
\hline 6 & Col. (Hazen) & $<15$ & $<15$ & $<15$ & $<15$ & $<15$ & $<15$ & $<15$ & $<15$ & $<15$ & $<15$ & $<15$ & $<15$ \\
\hline 7 & Turb. (NTU) & 10.70 & 7.91 & 11.65 & 6.12 & 11.65 & 23.91 & 12.40 & 9.33 & 27.45 & 6.55 & 8.87 & 10.73 \\
\hline 8 & THH (mg/L) & 87.75 & 90.00 & 40.65 & 112.0 & 40.65 & 120.0 & 75.0 & 56.0 & 76.0 & 56.0 & 79.0 & 47.0 \\
\hline 9 & Ca. Hard $(\mathrm{mg} / \mathrm{L})$ & 68.0 & 38.0 & 20.0 & 19.0 & 20.0 & 22.0 & 80.0 & 52.0 & 36.0 & 16.0 & 19.5 & 114.0 \\
\hline 10 & Mg. Hard (mg/L) & 19.0 & 24.0 & 16.0 & 48.0 & 16.0 & 10.0 & 16.0 & 68.0 & 41.0 & 18.0 & 31.0 & 11.0 \\
\hline 11 & $\mathrm{NO}_{3}{ }^{2-}(\mathrm{mg} / \mathrm{L})$ & 5.7 & 7.4 & 6.8 & 8.3 & 6.8 & 7.1 & 12.9 & 8.8 & 7.6 & 8.3 & 6.4 & 7.6 \\
\hline 12 & $\mathrm{Cl}(\mathrm{mg} / \mathrm{L})$ & 85.5 & 112.0 & 12.7 & 54.7 & 12.7 & 18.9 & 0.013 & 12.5 & 49.3 & 17.3 & 13.5 & 37.0 \\
\hline 13 & $\mathrm{Mn}(\mathrm{mg} / \mathrm{L})$ & 0.012 & 0.008 & 0.023 & 0.017 & 0.023 & 0.033 & 0.018 & 0.013 & 0.015 & 0.023 & 0.018 & 0.009 \\
\hline 14 & OD (Unobj) & Unobj & Unobj & Unobj & Unobj & Unobj & Unobj & Unobj & Unobj & Unobj & Unobj & Unobj & Unob \\
\hline 15 & $\mathrm{TA}(\mathrm{mg} / \mathrm{L})$ & 98.6 & 100.9 & 102.7 & 97.0 & 102.7 & 88.7 & $100.5^{\circ}$ & 103.0 & 79.0 & 86.3 & 110.0 & 108.1 \\
\hline 16 & $\left.\mathrm{~S}_{4}{ }^{2-}\right)(\mathrm{mg} / \mathrm{L})$ & 118.9 & 104.5 & 109.7 & 113.9 & 109.7 & 110.6 & 99.0 & 118.0 & 101.0 & 102.8 & 125.0 & 97.0 \\
\hline 17 & $\mathrm{Fe}(\mathrm{mg} / \mathrm{L})$ & 0.02 & 0.13 & 0.16 & 0.09 & 0.16 & ND & 0.09 & 0.07 & N.D & 0.02 & 4.6 & 4.3 \\
\hline 18 & $\mathrm{Na}(\mathrm{mg} / \mathrm{L})$ & 40.0 & 73.0 & 36.0 & 18.6 & 36.0 & 68.0 & 57.0 & 81.5 & 77.6 & 54.7 & 78.9 & 91.7 \\
\hline 19 & $\mathrm{HCO}_{3}{ }^{2-}(\mathrm{mg} / \mathrm{L})$ & 86.0 & 57.0 & 43.9 & 56.0 & 43.9 & 41.3 & 62.0 & 41.8 & 66.2 & 35.0 & 28.7 & 48.6 \\
\hline 20 & $\mathrm{Ca}(\mathrm{mg} / \mathrm{L})$ & 37.7 & 16.7 & 9.7 & 31.5 & 9.7 & 7.6 & 6.7 & 8.9 & 17.6 & 40.0 & 9.1 & 7.8 \\
\hline 21 & $\mathrm{Mg}(\mathrm{mg} / \mathrm{L})$ & 8.3 & 3.9 & 5.9 & 7.4 & 5.9 & 4.1 & 9.0 & 6.8 & 4.1 & 2.9 & 3.8 & 2.2 \\
\hline 22 & TDS $(\mathrm{mg} / \mathrm{L})$ & 113.2 & 43.1 & 23.0 & 17.5 & 23.0 & 17.1 & 12.8 & 33.9 & 10.6 & 14.6 & 55.0 & 32.5 \\
\hline 23 & $\mathrm{SS}(\mathrm{mg} / \mathrm{L})$ & 31.4 & 11.8 & 31.7 & 11.9 & 31.7 & 18.7 & 75.4 & 66.0 & 19.8 & 23.1 & 34.7 & 13.5 \\
\hline 24 & TC Cfu/0.ml & 23 & 17 & 20 & 15 & 20 & 19 & 28 & 26 & 40 & 52 & 45 & 26 \\
\hline 25 & E-coli Cfu/100ml & 0 & 0 & 1 & 1 & 1 & 1 & 1 & 1 & 1 & 0 & 0 & 0 \\
\hline
\end{tabular}
solids; $\mathrm{TA}=$ total alkalinity; $\mathrm{SS}=$ suspended solids; $\mathrm{TC}=$ total coliform

Determination of coliform levels: Multiple tube fermentation was used. All apparatus were first sterilized using autoclave. Series of dilutions were made up to $1 \mathrm{ft}$ each of the 10-2 sample test was poured inside the sterilizer and covered with Petridish. Some portion of the nutrient that had already 
been prepared was added. The mixture was gently and evenly rocked and allowed to gel (solidify), It was then incubated for three (3) days after which it was transferred to the refrigerator. The water sample was cultured, single and double strength MacConkey broth in the tubes showing positive result, i.e, medium turned yellow or pale yellow and formation of gas in the Durham tubes were further cultured using eosine methylene blue. Colonies of green metallic sheen or eosin methylene blue confirmed the presence of E-coli in such water sample.

\section{RESULT AND DISCUSSION}

Temperature: The temperatures of the samples are somehow uniform and found to vary between $27^{\circ} \mathrm{c}$ and $29^{\circ} \mathrm{C}$. The conditions of the wells have some influence on the temperature e.g. the depth of well, whether or not the well is covered and the material used for the cover etc.
Colour: From the visual observation of the water samples, most of the samples appeared colored due to the host rock and the dissolved materials.

Odour and Taste: The well water samples collected were subjected to mammal sensation. All the samples were found not to be objectionable to man.

Total Suspended Solids (TSS): Solid matter or residue suspended in water may affect its quality adversely in a number of ways. Water with high residues is less acceptable for consumption, for domestic and industrial purposes as it might induce unfavourable physiological reaction. According to Ademoroti (1996), the amount of suspended solid in water can vary widely, depending on flow, season and handling. The amount of suspended solid in all the well water samples tested varies from $11.8 \mathrm{mg} / \mathrm{L}$ to $75.4 \mathrm{mg} / \mathrm{L}$. These values are low compared to WHO permissible range for suspended solid which is about $500 \mathrm{mg} / \mathrm{L}$. This may be as a result of the filtration capacity of the weathered materials overlying the bed rock in the study area.

\begin{tabular}{|c|c|c|c|c|c|c|c|c|c|}
\hline $\mathbf{S} / \mathbf{N}$ & Parameter/Unit & W13 & W14 & W15 & W16 & W17 & W18 & W19 & W20 \\
\hline 1 & APP (CLEAR) & 23.65 & 14.43 & 10 & 4.4 & 0 & 0 & 0 & 0.52 \\
\hline 2 & DWW (m) & 2.80 & 2.60 & 2.40 & 2.40 & 2.50 & 2.90 & 2.30 & 2.30 \\
\hline 3 & Temp. (NS) & 25.25 & 4.86 & 18 & 8.0 & 0.01 & 0 & 0 & 1.27 \\
\hline 4 & Cond. $(\mu \mathrm{s} / \mathrm{cm})$ & 36.07 & 20.9 & 27 & 7.5 & 0 & 0 & 0 & 0.85 \\
\hline 5 & $\mathrm{pH}$ & 35.27 & 2.67 & 23 & 4.6 & 0.01 & 0 & 0 & 0.21 \\
\hline 6 & Col. (Hazen) & 30.06 & 20.41 & 13 & 2.6 & 0.2 & 0 & 0 & 1.78 \\
\hline 7 & Turb. (NTU) & 16.43 & 3.65 & 11 & 5.1 & 0.01 & 0 & 0 & 1.88 \\
\hline 8 & THH (mg/L) & 2.00 & 0.49 & 17 & 4.9 & 0 & 0 & 0 & 1.10 \\
\hline 9 & Ca. Hard (mg/L) & 11.62 & 3.16 & 24 & 29.3 & 0.01 & 0 & 0 & 0.29 \\
\hline 10 & Mg. Hard (mg/L) & 11.22 & 0.97 & 9.5 & 4.2 & 0.1 & 0 & 0 & 1.82 \\
\hline 11 & $\mathrm{NO}_{3}{ }^{2-}(\mathrm{mg} / \mathrm{L})$ & 11.62 & 7.29 & 18 & 7.0 & 0.01 & 0 & 0 & 0.27 \\
\hline 12 & $\mathrm{Cl}(\mathrm{mg} / \mathrm{L})$ & 24.85 & 8.75 & 23 & 14.1 & 0.06 & 0 & 0 & 0.05 \\
\hline 13 & $\mathrm{Mn}(\mathrm{mg} / \mathrm{L})$ & 27.66 & 13.12 & 22.5 & 8.8 & 0.04 & 0 & 0 & 0.67 \\
\hline 14 & OD (Unobj) & 19.24 & 44.96 & 40 & 6.5 & 0.04 & 0 & 0 & 1.28 \\
\hline 15 & $\mathrm{TA}(\mathrm{mg} / \mathrm{L})$ & 15.63 & 13.85 & 12.5 & 5.0 & 0.02 & 0 & 0 & 0.05 \\
\hline 16 & $\left.\mathrm{SO}_{4}{ }^{2-}\right)(\mathrm{mg} / \mathrm{L})$ & 18.84 & 43.25 & 36.5 & 26 & 0.03 & 0 & 0 & 0.15 \\
\hline 17 & $\mathrm{Fe}(\mathrm{mg} / \mathrm{L})$ & 5.0 & 5.2 & 53 & 0.08 & 0.05 & 0 & 0 & 0.1 \\
\hline 18 & $\mathrm{Na}(\mathrm{mg} / \mathrm{L})$ & 28.06 & 41.8 & 42 & 2.7 & 0.01 & 0 & 0 & 0.25 \\
\hline 19 & $\mathrm{HCO}_{3}{ }^{2-}(\mathrm{mg} / \mathrm{L})$ & 16.03 & 43.74 & 27 & 21.6 & 0.03 & 0 & 0 & 0.95 \\
\hline 20 & $\mathrm{Ca}(\mathrm{mg} / \mathrm{L})$ & 11.62 & 30.86 & 27 & 18.6 & 0.15 & 0 & 0 & 0.56 \\
\hline 21 & $\mathrm{Mg}(\mathrm{mg} / \mathrm{L})$ & 25.65 & 39.85 & 18 & 8.0 & 0.05 & 0 & 0 & 0.45 \\
\hline 22 & TDS $(\mathrm{mg} / \mathrm{L})$ & 12.82 & 45.93 & 46 & 6.1 & 0.30 & 0 & 0 & 1.87 \\
\hline 23 & $\mathrm{SS}(\mathrm{mg} / \mathrm{L})$ & 40.88 & 32.08 & 43 & 2.9 & 0 & 0 & 0 & 0.06 \\
\hline 24 & $\mathrm{TC} \mathrm{Cfu} / 0 . \mathrm{ml}$ & 13.23 & 47.87 & 43.5 & 5.5 & 0.01 & 0 & 0 & 0.97 \\
\hline 25 & E-coli Cfu/100ml & 9.22 & 9.48 & 18 & 5.5 & 0 & 0 & 0 & 1.20 \\
\hline
\end{tabular}

\begin{tabular}{|c|c|c|c|c|c|c|c|c|c|c|c|}
\hline $\mathbf{S} / \mathbf{N}$ & Sample & $\mathbf{C u}$ & $\mathbf{P b}$ & Zn & $\mathbf{K}$ & V & Cd & $\mathrm{Cr}$ & $\mathrm{Ni}$ & $\mathbf{B a}$ & $\mathrm{Fe}$ \\
\hline 1. & Well 1 & 0.3 & 0.009 & 11.3 & 97.5 & 0.1 & 0.1 & 0.9 & 0.2 & 0.4 & 0.02 \\
\hline 2. & Well 2 & 0.3 & 0.005 & 5.1 & 96.3 & 0.1 & 0.1 & 0.7 & 0.2 & 0.4 & 0.13 \\
\hline 3. & Well 3 & 0.2 & 0.07 & 8.4 & 135.1 & - & 0.1 & 0.5 & 0.1 & 0.5 & 0.16 \\
\hline 4. & Well 4 & 0.4 & 0.08 & 5.5 & 140.5 & - & 0.1 & 0.3 & 0.1 & 0.40 & 0.09 \\
\hline 5. & Well 5 & 0.1 & 0.006 & 3.9 & 111.9 & - & 0.002 & 0.2 & 0.1 & 0.1 & 0.16 \\
\hline 6. & Well 6 & 0.2 & - & 4.6 & 131.2 & 0.1 & 0.001 & 0.4 & - & 0.2 & - \\
\hline 7. & Well 7 & 0.2 & 0.01 & 9.5 & 115.3 & - & - & 0.3 & - & 0.4 & 0.09 \\
\hline 8. & Well 8 & 0.2 & 0.01 & 7.9 & 120.5 & 0.1 & - & 0.4 & 0.3 & 0.3 & 0.07 \\
\hline 9. & Well 9 & 0.1 & 0.01 & 0.9 & 120.5 & - & - & 0.6 & 0.1 & 0.3 & - \\
\hline 10. & Well 10 & 0.1 & 0.008 & 5.1 & 110.5 & - & 0.001 & 0.2 & 0.1 & 0.5 & 0.02 \\
\hline 11. & Well 11 & 0.1 & 0.01 & 5 & 110.0 & 0.1 & 0.0004 & 0.05 & 0.1 & 0.05 & 4.6 \\
\hline 12. & Well 12 & 0.1 & 0.01 & 5 & 110.0 & - & 0.0003 & 0.05 & 0.1 & 0.05 & 4.3 \\
\hline 13. & Well 13 & 0.2 & 0.005 & 6.5 & 98.5 & - & - & 0.8 & - & 0.1 & 5.0 \\
\hline 14. & Well 14 & 0.2 & - & 7.8 & 120.0 & 0.1 & 0.0005 & 0.07 & - & 0.07 & 5.2 \\
\hline 15. & Well 15 & 0.4 & 0.01 & 5.9 & 113.9 & 0.1 & 0.0006 & 0.3 & 0.2 & 0.2 & 5.3 \\
\hline 16. & Well 16 & 0.1 & 0.002 & 9.0 & 139.5 & 0.1 & - & 0.6 & 0.3 & 0.09 & 0.08 \\
\hline 17. & Well 17 & 0.3 & 0.003 & 5.7 & 97.6 & - & 0.003 & 0.2 & 0.1 & 0.5 & 0.05 \\
\hline 18. & Well 18 & 0.1 & 0.009 & 8.0 & 95.8 & - & 0.003 & 0.9 & 0.1 & 0.4 & 0 \\
\hline 19. & Well 19 & 0.2 & 0.01 & 7.9 & 110.0 & 0.1 & 0.003 & 0.05 & - & 0.4 & 0 \\
\hline 20. & Well 20 & 0.3 & 0.004 & 8.5 & 125.3 & 0.1 & 0.003 & 0.5 & 0.2 & 0.1 & 0.1 \\
\hline 21. & Eva Water & 0.001 & 0 & 0.283 & 0 & 0 & 0 & 0 & 0 & 0 & 0.002 \\
\hline 22 & NAFDAC & 1.0 & 0.01 & 5.0 & 10.0 & 0 & 0.003 & 0.056 & 0 & 0.05 & 0.3 \\
\hline 23 & WHO & 2.0 & 0.01 & 3.0 & - & 0 & 0.003 & 0.05 & $1-2 \mu \mathrm{g} / \mathrm{L}$ & 0.07 & 1.0 \\
\hline
\end{tabular}


Table 4: Result of Physical Chemical Analysis

\begin{tabular}{|c|c|c|c|c|c|c|c|}
\hline Sample & $\begin{array}{l}\text { Turbidity } \\
\text { (NTU) }\end{array}$ & $\begin{array}{l}\text { Total } \\
\text { alkalinity } \\
(\mathrm{mg} / \mathrm{L})\end{array}$ & $\begin{array}{l}\text { Nitrate } \\
\mathrm{NO}_{3}^{2-} \\
(\mathrm{mg} / \mathrm{L})\end{array}$ & $\begin{array}{l}\text { Chloride } \\
\mathrm{Cr}^{-} \\
(\mathrm{mg} / \mathrm{L})\end{array}$ & $\begin{array}{l}\text { Sulphate } \\
\mathrm{SO}^{2} \\
(\mathrm{mg} / \mathrm{L})\end{array}$ & $\begin{array}{l}\text { Sodium } \\
\mathrm{Na}^{-} \\
(\mathrm{mg} / \mathrm{L})\end{array}$ & $\begin{array}{l}\text { Cakium } \mathrm{Ca}^{\top} \\
(\mathrm{mg} / \mathrm{L})\end{array}$ \\
\hline Well 1 & 10.70 & 98.6 & 5.7 & 85.5 & 118.9 & 40.0 & 37.7 \\
\hline Well 2 & 7.91 & 100.9 & 7.4 & 112.0 & 104.5 & 73.0 & 16.7 \\
\hline Well 3 & 11.65 & 102.7 & 6.8 & 12.7 & 109.7 & 36.0 & 9.7 \\
\hline Well 4 & 6.12 & 97.0 & 8.3 & 54.7 & 113.9 & 18.6 & 31.5 \\
\hline Well 5 & 11.65 & 102.7 & 6.8 & 12.7 & 109.7 & 36.0 & 9.7 \\
\hline Well 6 & 23.91 & 88.7 & 7.1 & 18.9 & 110.6 & 68.0 & 7.6 \\
\hline Well 7 & 12.40 & 100.5 & 12.9 & 0.013 & 99.0 & 57.0 & 6.7 \\
\hline Well 8 & 9.33 & 103.0 & 8.8 & 12.5 & 118.0 & 81.5 & 8.9 \\
\hline Well 9 & 27.45 & 79.0 & 7.6 & 49.3 & 101.0 & 77.6 & 17.6 \\
\hline Well 10 & 6.55 & 86.3 & 8.3 & 17.3 & 102.8 & 54.7 & 40.0 \\
\hline Well 11 & 8.87 & 110.0 & 6.4 & 13.5 & 125.0 & 78.9 & 9.1 \\
\hline Well 12 & 10.73 & 108.1 & 7.6 & 37.0 & 97.0 & 91.7 & 7.8 \\
\hline Well 13 & 16.43 & 15.63 & 11.62 & 24.85 & 18.84 & 28.06 & 11.62 \\
\hline Well 14 & 3.65 & 13.85 & 7.29 & 8.75 & 43.25 & 41.80 & 30.86 \\
\hline Well 15 & 11 & 12.5 & 18 & 23 & 36.5 & 42.0 & 6.27 \\
\hline Well 16 & 5.1 & 5.0 & 7.0 & 14.1 & 26 & 2.70 & 18.6 \\
\hline Well 17 & 0.01 & 0.02 & 0.01 & 0.06 & 0.03 & 0.01 & 0.15 \\
\hline Well 18 & 0 & 0 & 0 & 0 & 0 & 0 & 0 \\
\hline Well 19 & 0 & 0 & 0 & 0 & 0 & 0 & 0 \\
\hline Well 20 & 1.88 & 0.05 & 0.27 & 0.05 & 0.15 & 0.25 & 0.56 \\
\hline Eva Water & 0 & 0.40 & 0.001 & 0.52 & 0.001 & 0.05 & 2.99 \\
\hline NAFDAC & 5.0 & 100 & 10 & 100 & 100 & 200 & 0.003 \\
\hline WHO & 5.0 & 100 & 10 & 200 & 250 & 200 & 0.003 \\
\hline
\end{tabular}

\begin{tabular}{|c|c|c|c|c|c|}
\hline \multirow{2}{*}{$\begin{array}{l}\mathbf{S} / \mathbf{N} \\
1 .\end{array}$} & \multirow{2}{*}{$\begin{array}{l}\text { Parameter } \\
\text { Colour }\end{array}$} & \multirow{2}{*}{$\begin{array}{c}\begin{array}{c}\text { NAFDAC } \\
\text { maximum limits }\end{array} \\
3.0 \mathrm{TCU}\end{array}$} & \multirow{2}{*}{\begin{tabular}{|l} 
SON standard \\
3.0TCU
\end{tabular}} & \multicolumn{2}{|c|}{ WHO STANDARDS } \\
\hline & & & & $3.0 \mathrm{TCU}$ & $15.0 \mathrm{TCU}$ \\
\hline 2. & Odour & Unobjectionable & Unobjectionable & Unobjectionable & Unobjectionable \\
\hline 3. & Taste & Unobjectionable & Unobjectionable & Unobjectionable & Unobjectionable \\
\hline 4. & $\mathrm{Ph}$ at $20^{\circ} \mathrm{C}$ & $6.50-8.5$ & $6.5-8.5$ & $7.0-8.9$ & $6.50-9.50$ \\
\hline 5. & Turbidity & $5.0 \mathrm{NTU}$ & $5.0 \mathrm{NTU}$ & $5.0 \mathrm{NTU}$ & $5.0 \mathrm{NTU}$ \\
\hline 6. & Conductivity & $1000(\mathrm{us} / \mathrm{cm})$ & $1000(\mathrm{us} / \mathrm{cm})$ & $900(\mathrm{us} / \mathrm{cm})$ & $1200(\mathrm{us} / \mathrm{cm})$ \\
\hline 7. & Total solids & $500 \mathrm{mg} / \mathrm{L}$ & $500 \mathrm{mg} / \mathrm{L}$ & $500 \mathrm{mg} / \mathrm{L}$ & $1500 \mathrm{mg} / \mathrm{L}$ \\
\hline 8. & Total Alkalinity & $100 \mathrm{mg} / \mathrm{L}$ & $100 \mathrm{mg} / \mathrm{L}$ & $100 \mathrm{mg} / \mathrm{L}$ & $100 \mathrm{mg} / \mathrm{L}$ \\
\hline 9. & Phenolphthalein & $100 \mathrm{mg} / \mathrm{L}$ & $100 \mathrm{mg} / \mathrm{L}$ & $100 \mathrm{mg} / \mathrm{L}$ & $100 \mathrm{mg} / \mathrm{L}$ \\
\hline 10. & Alkalinity & - & - & - & - \\
\hline 11. & Chloride & $100 \mathrm{mg} / \mathrm{L}$ & $100 \mathrm{mg} / \mathrm{L}$ & $200 \mathrm{mg} / \mathrm{L}$ & $250 \mathrm{mg} / \mathrm{L}$ \\
\hline 12. & Fluoride & $1.0 \mathrm{mg} / \mathrm{L}$ & $1.0 \mathrm{mg} / \mathrm{L}$ & $1.0 \mathrm{mg} / \mathrm{L}$ & $1.5 \mathrm{mg} / \mathrm{L}$ \\
\hline 13. & Copper & $1.0 \mathrm{mg} / \mathrm{L}$ & $1.0 \mathrm{mg} / \mathrm{L}$ & $0.5 \mathrm{mg} / \mathrm{L}$ & $2.0 \mathrm{mg} / \mathrm{L}$ \\
\hline 14. & Iron & $0.3 \mathrm{mg} / \mathrm{L}$ & $0.3 \mathrm{mg} / \mathrm{L}$ & $1 \mathrm{mg} / \mathrm{L}$ & $3 \mathrm{mg} / \mathrm{L}$ \\
\hline 15. & Nitrate & $10 \mathrm{mg} / \mathrm{L}$ & $10 \mathrm{mg} / \mathrm{L}$ & $10 \mathrm{mg} / \mathrm{L}$ & $50 \mathrm{mg} / \mathrm{L}$ \\
\hline 16. & Nitrite & $0.02 \mathrm{mg} / \mathrm{L}$ & $0.02 \mathrm{mg} / \mathrm{L}$ & $0.2 \mathrm{mg} / \mathrm{L}$ & $3 \mathrm{mg} / \mathrm{L}$ \\
\hline 17. & Manganese & $2.0 \mathrm{mg} / \mathrm{L}$ & $0.05 \mathrm{mg} / \mathrm{L}$ & $0.1 \mathrm{nig} / \mathrm{L}$ & $0.4 \mathrm{mg} / \mathrm{L}$ \\
\hline 18. & Magnesium & $20 \mathrm{mg} / \mathrm{L}$ & $0.20 \mathrm{mg} / \mathrm{L}$ & 20 & $20 \mathrm{mg} / \mathrm{L}$ \\
\hline 19. & Zinc & $5.0 \mathrm{mg} / \mathrm{L}$ & $5.0 \mathrm{mg} / \mathrm{L}$ & $0.01 \mathrm{mg} / \mathrm{L}$ & $3.0 \mathrm{mg} / \mathrm{L}$ \\
\hline 20. & Selenium & $0.01 \mathrm{mg} / \mathrm{L}$ & NS & $0.01 \mathrm{mg} / \mathrm{L}$ & $0.01 \mathrm{mg} / \mathrm{L}$ \\
\hline 21. & Silver & - & - & NS & NS \\
\hline 22. & Cyanide & $0.01 \mathrm{mg} / \mathrm{L}$ & $0.01 \mathrm{mg} / \mathrm{L}$ & $0.01 \mathrm{mg} / \mathrm{L}$ & $0.07 \mathrm{mg} / \mathrm{L}$ \\
\hline 23. & Sulphate & $100 \mathrm{mg} / \mathrm{L}$ & $100 \mathrm{mg} / \mathrm{L}$ & $250 \mathrm{mg} / \mathrm{L}$ & $500 \mathrm{nig} / \mathrm{L}$ \\
\hline 24. & Calcium & $75 \mathrm{mg} / \mathrm{L}$ & $75 \mathrm{mg} / \mathrm{L}$ & NS & NS \\
\hline 25. & Aluminum & $0.5 \mathrm{mg} / \mathrm{L}$ & NS & $0.2 \mathrm{mg} / \mathrm{L}$ & $0.2 \mathrm{mg} / \mathrm{L}$ \\
\hline 26. & Potassium & $10.0 \mathrm{mg} / \mathrm{L}$ & $10 \mathrm{mg} / \mathrm{L}$ & NS & NS \\
\hline 27. & Lead & $0.01 \mathrm{mg} / \mathrm{L}$ & $0.01 \mathrm{mg} / \mathrm{L}$ & $0.01 \mathrm{mg} / \mathrm{L}$ & $0.01 \mathrm{mg} / \mathrm{L}$ \\
\hline 28. & Chromium & $0.05 \mathrm{mg} / \mathrm{L}$ & $0.05 \mathrm{mg} / \mathrm{L}$ & $0.05 \mathrm{mg} / \mathrm{L}$ & $0.05 \mathrm{mg} / \mathrm{L}$ \\
\hline 29. & Cadmium & $0.003 \mathrm{mg} / \mathrm{L}$ & $0.03 \mathrm{mg} / \mathrm{L}$ & $0.003 \mathrm{mg} / \mathrm{L}$ & $0.003 \mathrm{mg} / \mathrm{L}$ \\
\hline 30. & Arsenic & $0.01 \mathrm{mg} / \mathrm{L}$ & $0.01 \mathrm{mg} / \mathrm{L}$ & $0.01 \mathrm{mg} / \mathrm{L}$ & $0.01 \mathrm{mg} / \mathrm{L}$ \\
\hline 31. & Barium & $0.05 \mathrm{mg} / \mathrm{L}$ & $0.05 \mathrm{mg} / \mathrm{L}$ & $0.05 \mathrm{mg} / \mathrm{L}$ & $0.07 \mathrm{mg} / \mathrm{L}$ \\
\hline 32. & Mercury & $0.001 \mathrm{mg} / \mathrm{L}$ & $0.001 \mathrm{mg} / \mathrm{L}$ & $0.01 \mathrm{mg} / \mathrm{L}$ & $0.001 \mathrm{mg} / \mathrm{L}$ \\
\hline 33. & Antimony & NS & NS & - & $0.02 \mathrm{mg} / \mathrm{L}$ \\
\hline 34. & Tin & - & - & - & $1-2 \mu \mathrm{g} / 1$ \\
\hline 35. & Nickel & - & - & & $0.02 \mathrm{mg} / \mathrm{L}$ \\
\hline 36. & Total hardness $\left(\mathrm{CaCO}_{3}\right)$ & $100 \mathrm{mg} / \mathrm{L}$ & $100 \mathrm{mg} / \mathrm{L}$ & $100 \mathrm{mg} / \mathrm{L}$ & $500 \mathrm{mg} / \mathrm{L}$ \\
\hline 37. & Vinyl chloride & $0 \mathrm{mg} / \mathrm{L}$ & $0 \mathrm{mg} / \mathrm{L}$ & $0 \mathrm{mg} / \mathrm{L}$ & $0.003 \mathrm{mg} / \mathrm{L}$ \\
\hline
\end{tabular}

$p H$ : The samples tested have below $7.8 \mathrm{pH}$, varying from 6.9-7.8 (i.e weakly acidic to slightly alkaline). This implies that there is little or no dissolved carbonate and hydroxide ions in the samples. This also indicates the presence of free carbon IV oxide.
Thus, the dissolved inorganic carbon exists almost entirely as bicarbonate ions.

Conductivity: The conductivity values for all the well water samples tested varies from $40-210 \mu / \Omega \mathrm{m}$. All the 
water tested were below those of the NAFDAC, SON and WHO standard for drinking water, which is indicative of relatively low electrical conductivity or dissolved ions.

Total Dissolved Solids (TDS): The total dissolved solid content is also known as the total salt concentration of groundwater. Groundwater can be classified according to its TDS content. Hem, (1970), classified water on the basis of TDS as stated in Table 6.

\begin{tabular}{ll}
\multicolumn{2}{c}{ Table 6: Classification of TDS in water } \\
\hline Classification & TDS range $(\mathbf{m g} / \mathbf{L})$ \\
\hline Fresh & $<1000$ \\
Moderately saline & $3000-10000$ \\
Very saline & $10000-35000$ \\
Brine & $>35000$ \\
\hline
\end{tabular}

The TDS concentration for all the well water samples varies from $10.6-113.2 \mathrm{mg} / \mathrm{L}$ they can therefore be classified as fresh water. The values also fall below the WHO desirable limit which is $500 \mathrm{mg} / \mathrm{L}$.

Total Hardness: The hardness of water relates to its reaction with soap and to the scale and encrustation accumulating in containers or conduits where water is heated. The WHO recommended standard for drinking water is $500 \mathrm{mg} / \mathrm{L}$. All the samples tested have hardness which falls below the WHO standard. All the samples tested possess carbonate and magnesium sulphate hardness. Hem (1970), classified water according to its hardness as presented in Table 7

\begin{tabular}{cl} 
Table 7: Classification of total hardness \\
\hline Hardness & Class $(\mathbf{m g} / \mathbf{L})$ \\
\hline Soft & $0-60$ \\
Moderate & $61-120$ \\
Hard & $121-180$ \\
Very hard & $>180$ \\
\hline
\end{tabular}

Based on the classification above, four (4) of the wells tested are grouped as soft, while eight (8) are moderately hard. Generally, the water sample values vary from $40.65-120 \mathrm{mg} / \mathrm{L}$ that is soft to moderately hard. Some amount of magnesium sulphate $\left(\mathrm{MgSO}_{4}\right)$ hardness is suspected due to the influence of silicate minerals in the basement rock.

Total Alkalinity: The results for total alkalinity show values ranging between $88-118.9 \mathrm{Mg} / \mathrm{L}$. wells $1,4,5,6,9$ to 15 are below the WHO standard which is 100 while wells $2,3,7,8,16$ to 20 are above the WHO standard. The wells that are below clearly indicate a relatively low amount of $\mathrm{CaCO}_{3}$ and $\mathrm{CO}_{2}$. Which are the controlling factors for alkalinity and that the sample are highly basic in nature.
Calcium $\left(\mathrm{Ca}^{2+}\right)$ : Calcium is one of the most abundant cat ions in the entire water sample tested with concentration varying from $6.7-40.0 \mathrm{mg} / \mathrm{L}$. All the samples tested have less than the NAFDAC maximum limit of $75 \mathrm{mg} / \mathrm{L}$. The presence of calcium in water has effect on uses of the water. Calcium generally combines with bicarbonate, carbonate, sulphur and silica to form heat retarding, pipe clogging, scales in boilers and other heat exchange equipment. Calcium combines with ions of fatty acid to form soap subs.

Magnesium $\left(\mathrm{Mg}^{2+}\right)$ : Magnesium concentration in the water samples varies from $2.2-9.0 \mathrm{mg} / \mathrm{L}$ as compared to the accepted maximum limit of WHO standard 20mg/L. Magnesium content in the samples are less than the $20 \mathrm{mg} / \mathrm{L}$ of NAFDAC standard. The major sources of magnesium in water are from igneous rocks and dolomite. High concentration of magnesium has a laxative effect, (Durfer and Baker, 1964).

Sodium $\left(\mathrm{Na}^{+}\right)$: The dissolved content values vary from $18.6-91.7 \mathrm{mg} / \mathrm{l}$. The WHO standard for sodium in drinking water is $200 \mathrm{mg} / \mathrm{l}$. All the water samples tested gave sodium value below $100 \mathrm{mg} / \mathrm{L}$. When sodium is greater than $50 \mathrm{mg} / \mathrm{l}$ in the presence of suspended matters, it causes foaming which accelerates scale formation and corrosion in boilers (Durfer and Baker, 1964).The major source of sodium is from feldspar (albite), clay minerals, evaporate and industrial waste.

Sulphate $\left(\mathrm{SO}_{4}{ }^{2-}\right)$ : The concentration of sulphate ion varies from $97-125 \mathrm{mg} / \mathrm{L}$ which fall below the maximum desirable limit of $250 \mathrm{mg} / \mathrm{L}$ for drinking water by WHO. Sulphate combines with calcium to form an adherent heat retarding scale. High concentration of sulphate gives better taste to water, and can produce laxative infects in human. It causes permanent hardness in water through the formation of magnesium sulphate. Sulphate is formed from the oxidation of pyrite and other sulphate widely distributed in igneous and sedimentary rocks.

Bicarbonate: The concentration of bicarbonate in all the samples varies from 28.7 - $86 \mathrm{mg} / \mathrm{L}$ which are relatively below $200 \mathrm{mg} / \mathrm{L}$ of the WHO standard limit. When heated, bicarbonate is changed into steam, $\mathrm{CO}_{2}$ and $\mathrm{CO}_{3}$. The carbonate combines with alkaline earth metals especially $\mathrm{Ca}$ and $\mathrm{Mg}$ to form a crust-like scale of $\mathrm{CaCo}_{3}$ that retards flow of heat through pipes. The major sources are limestone, dolomite less than $50 \mathrm{mg} / \mathrm{L}$ in water. 
Nitrate $\left(\mathrm{NO}_{3}\right)$ : Nitrates are produced naturally as part of the nitrogen cycle. When a bacterial production line breaks down, toxic ammonia wastes first forms nitrite and then oxidizes into nitrate. These bacteria are called nitrogen fixing bacteria, for example, nitrosomonas and nitrobacter. Upon the death and decay of plants and bacteria, the nitrates are leached from the topsoil into the groundwater by infiltration (Durfer and Baker, 1964). The dissolved nitrate concentration in Ikiran-Ile water system varies from 5.7-9.1mg/L. The maximum desired limit of nitrate in drinking water by WHO is $10 \mathrm{mg} / \mathrm{L}$. All the well water samples tested gave values below this limit. Nitrates also gets into groundwater from lawn fertilizer, run off, leaking tanks, septic discharge, and animal waste. Nitrates stimulate the growth of planktons and waterweeds that provide food for fishes. However, if algae grow too widely, oxygen level will be reduced and fishes will die. Higher concentration of nitrate in drinking water is harmful to infant as a result of methaemoglobin or blue baby disease because young infants lack sufficient acidity, nitrate reducing bacteria. This association may be as a result of pollution by sewage still undergoing nitrification.

Lead $(\mathrm{Pb})$ : The result of the analysis indicates high value of lead in two samples W3 and W4 with values $0.07 \mathrm{mg} / \mathrm{L}$ and $0.08 \mathrm{mg} / \mathrm{L}$ respectively in contrast to other wells whose lead values were within the range of WHO standard which is $0.01 \mathrm{mg} /$. This high lead values are unique to samples 3 and 4 considering the likely source of lead in the wells' waters to have been from vehicular motor tyres that were used to line the two wells which were not found in the other wells. The effect of the high concentration of lead, as analyzed from these sample will be, affecting red blood cell chemistry, delay in normal physical growth in children and can also cause slight increase in blood pressure in some adults.

Zinc $(\mathrm{Zn})$ : The concentration of zinc from the analysis of the water samples indicate values ranging between $0.9-11.3 \mathrm{mg} / \mathrm{L}$ which exceed the WHO's maximum desirable limit of $0.01 \mathrm{mg} / \mathrm{L}$ and highest permissible limit of $3.0 \mathrm{mg} / \mathrm{L}$. NAFDAC and SON limit of $5.0 \mathrm{mg} / \mathrm{L}$ was also exceeded by most water samples. The high concentration of zinc can be attributed to run-off from industrial waste and most times occurring naturally in groundwater. The presence of zinc aids in the healing of wounds and generally causes no ill effect. However excess of it gives taste to water and also becomes toxic to plants.

Copper $(\mathrm{Cu})$ : The concentration of copper in the samples analyzed shows values ranging from 0.1 $0.4 \mathrm{mg} / \mathrm{L}$. the values fall below the WHO desirable limit of $0.5 \mathrm{mg} / \mathrm{L}$. All the values also fall below the NAFDAC and SON's standard limit of $1.0 \mathrm{mg} / \mathrm{L}$ making the water safe for consumption. In the situation where the standard is exceeded, it can result in stomach and intestinal disorder, liver, damage and anemia.

Chromium $(\mathrm{Cr})$ : From the analysis, the values for the degree of concentration of chromium in the water samples from the wells ranges from $0.05-0.9 \mathrm{mg} / \mathrm{L}$; this value exceeds the WHO standard of $0.05 \mathrm{mg} / \mathrm{L}$ as well as NAFDAC and SON's standard limit of $0.05 \mathrm{mg} / \mathrm{L}$ of which wells 11 and 12 were the same with WHO standard The likely source of chromium in groundwater include run-off from abandoned mining operations, fossil fuel combustion and waste incineration. The consequences of over concentration of chromium in drinking water include liver and kidney damage, respiratory damage and internal hemorrhage.

Nickel $(\mathrm{Ni})$ :From the result of the analysis of the water samples, it was seen that the value for the concentration of nickel ranges from $0.1-0.3 \mathrm{mg} / \mathrm{L}$ for ten wells while it is non-detectable (ND) in two (wells 6 and 7). WHO maximum permissible limit for nickel in groundwater is $0.02 \mathrm{mg} / \mathrm{L}$. Comparing values from the analysis with WHO standard shows clearly that the range of values from the analysis exceed the WHO standard limit, thus making the water unsafe for human consumption. The effects resulting from high concentration of nickel in water include damages to heart and liver.

Barium ( $\mathrm{Ba}$ ): The concentration of barium in the twenty (20) samples analyzed indicates values ranging between $0.05-0.4 \mathrm{mg} / \mathrm{L}$. This value exceeds WHO maximum permissible limit of $0.07 \mathrm{mg} / \mathrm{L}$ thus making the water unsafe for consumption. High concentration of barium causes a variety of cardiac, gastro-intestinal and neuromuscular effect associated with hypertension.

Cadmium $(C d)$ : WHO, NAFDAC and SON have a uniform standard limit $0.003 \mathrm{mg} / \mathrm{L}$ for cadmium in drinking water. Result from the heavy mineral analysis of samples from the twenty (20) wells gave a uniform value of $0.1 \mathrm{mg} / \mathrm{L}$ for four (4) wells (i.e wells $1,2,3,4)$ and 0.003 in four (4) wells $(17,18,19,20)$ and below standard in seven (7) wells $(5,6,10,11,12,14,15)$ and completely Non detectable in the other five (5) wells. The values obtained from the four wells have exceeded the standard stated above which of course poses a serious health risk when consumed. Over concentration of cadmium in water causes high blood pressure, destroys testicular 
tissue and red blood cells. It also becomes toxic to aquatic biota.

Biological Analysis:Biological analysis is important for detecting bacteria and pathogenic (diseasecausing) organisms in water. The biological quality of groundwater in all the wells is high. The total bacteria count in the groundwater samples varies from 17$52 \mathrm{Cfu} / 0.1 \mathrm{ml}$. The E-coli count also varies from 0$1 \mathrm{Cfu} / 100 \mathrm{ml}$. The coliform bacteria and their associates live on ground surface and in areas exposed to sewage disposal, dumping of human and other animal faeces. Coliform bacteria can be found in shallow wells with high water table or wells very close to sewage tanks, pit latrine and other sources of pollution. The effects of these bacteria include diseases like diarrhoea, gastroenteritis, catarrh and infection of the urinary tract, if consumption persists. The variation of these parameters in the study area is as a result of human influence, for example, domestic uses, disposal of waste and agricultural practices. Also, natural activities may affect the quality of groundwater for example, leaching of soil particles during filtration, decay of organic matter, precipitation, salt-water intrusion and exchange of ions within bed rock (Davis and Dewiest,(1966).

Control Sample:The control sample is sample 21 which is Eva bottled drinking water, from all the analyses carried out on this sample it is obvious that the water is safe for drinking since it satisfies the requirements of both NAFDAC and WHO for any safe drinking water. All the parameters tested for were within the limits of NAFDAC and WHO.

Conclusion: This study has been able to enhance sustainable water development through the various analyses that was carried out. Knowledge gained from the analysis of the harmful effects of some of the heavy metals found in the water, plus the recommendations given will go a long way in safe guarding the health of inhabitants of the study area in particular, and Nigeria in general.

Acknowledgment: The authors are grateful to Prof. W. O. Emofurieta for technical support during the course of this study.

\section{REFERENCES}

Ademoroti, CMA (1996). Environmental Chemistry and Toxicology flouted Press Limited. Ibadan First Edition, page 171- 182.

Akujieze, CN (1999). Groundwater Quality in Benin Urban of the Oligocene- Pleistocene Benin Formation, a paper presented at $12^{\text {th }}$ Annual National Conference of Nigerian Association of Hydrogeologists.

Davis, SN; Dewiest, RJM (1966).Hydrogeology, John Wiley and Sons, New York. 463pp.

Durfer CN, Baker, F (1964). Public water supplies of the 10 larger city in the U.S. Geological Survey. Water Supply, 1812: 364.

Hem, JO (1970).Interpretation of the Chemical Characteristic of Natural Water. Geological Survey Water Supply Paper $3^{\text {rd }}$ Edition U.S.A Paper Number 1473, Pg363-400

Oloto, IN: Anyanwu, DE (2013). Petrology of IbilloMangongo area of Igarra, Edo State, Nigeria. Adv. Appl. Sci. Res., 2013, 4(3):140-145

Rahaman, MA (1976). Review of the Basement Geology of SW Nigeria in Geology of Nigeria. Elizabethan Publishing Company, Nigeria. pp. 41-58.

Walton, WC (1970). Groundwater Resource Evaluation. McGraw Hill Book Co., New York, 664 pp. 